\title{
Research on Mass Ratio in Secondary Vibration Isolation
}

\author{
Hao $\mathrm{Wu}^{1, \mathrm{a}^{*}}$, Min $\mathrm{Chen}^{2, \mathrm{~b}}$ \\ (1. Liaoning Economic Vocational Technological Institute, Shenyang, Liaoning, 110122,China; \\ 2. Fushun Bo Yu Noise Control and Research Co., Ltd, Fushun, Liaoning, 113006,China) \\ a email address: wutietong@163.com, bemail: 995800417@qq.com
}

KeyWords: secondary vibration isolation, vibration isolation frame, mass ratio, resonance frequency, sound transmission in solid, spring

Abstract: The secondary vibration isolation may be used to abate sound transmission in solid in the application of damping and noise reduction treatment engineering, which can have an ideal effect. The secondary vibration isolation system has a natural low frequency so it can isolate low frequency vibration. The choice of vibration isolation frame mass or ratio of vibration isolation frame mass and unit mass is key technology. Resonant mass ratio range may be abtained by way of research on the relationship between frequency ratio and mass ratio. The relationship between vibration isolation efficiency and mass ratio may be figured out through the example. The ratio of vibration isolation frame mass in secondary vibration isolation affects system's resonance frequency. Resonance frequency can be escaped when mass ratio is above 0.3. Better efficiency can be gained through big mass ratio when necessary.

\section{Preface}

At present, the application of the secondary vibration isolation technology increased in noise reduction treatment project ${ }^{[1-2]}$. From January 1,2008, Noise Emission Standard for Social \& Living Environment (GB22337-2008) of national standard of PRC began to be carried out. Secondary vibration isolation can satisfy the stricter standard for sound transmission in solid. The choice of vibration isolation frame mass or ratio of vibration isolation frame mass and unit mass is key technology. In this thesis, the influence of vibration isolation frame mass or ratio of vibration isolation frame mass on vibration isolation parameter will be discussed.

\section{Rational Option of Mass or Ratio of Vibration Isolation Frame Mass}

On the design of secondary vibration isolation, $\mathrm{m}_{2}$, which is mass of vibration isolation frame, $\mathrm{K}_{1}$ and $\mathrm{K}_{2}$, which are spring stiffness of secondary vibration isolation, are the dynamic parameters to be determined. While, $\mathrm{K}_{1}$ can be designed as first vibration isolation and $\mathrm{K}_{2}$ can be devised according to the consistant deformation between the two levels ${ }^{[3]}$. Then, how to choose mass $\mathrm{m}_{2}$ or how to determine mass ratio $\mu$ matters.

\section{Fundamental Formula}

According to the theory of secondary vibration isolation ${ }^{[4-5]}$, the following conclusion can be achieved: 


$$
\begin{aligned}
& \left.\omega_{1}=\sqrt{\frac{\omega_{n}^{2}}{2 \mu}\left[(s+\mu+1) \pm \sqrt{(s+\mu+1)^{2}-4 s \mu}\right.}\right] \\
& \omega_{2} \\
& T_{f}=\frac{\omega_{n}^{4}}{\left(\omega^{2}-\omega_{1}^{2}\right)\left(\omega^{2}-\omega_{2}^{2}\right) \mu} \times \frac{k_{2}}{K_{1}}
\end{aligned}
$$

in the formula, $\mu=\mathrm{m}_{2} / \mathrm{m}_{1}$, mass ratio; $\mathrm{S}=\frac{K_{2}}{K_{1}}$, stiffness ratio; $\omega_{n}=\sqrt{\frac{k_{1}}{m_{1}}}$, intrinsic frequency.

Suppose the spring stiffness deformation is opted in both the first and secondary vibration

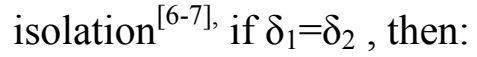

$\mathrm{K}_{2}=(1+\mu) \mathrm{K}_{1}$

Put (3) into two intrinsic frequency (1), then get:

$$
\begin{gathered}
\omega_{1}^{2}=\omega_{n}^{2} \frac{1+\mu+\sqrt{1+\mu}}{\mu} \\
\omega_{2}^{2}=\omega_{n}^{2} \frac{1+\mu-\sqrt{1+\mu}}{\mu}
\end{gathered}
$$

\section{Relationship of Mass Ratio and Frequency Ratio}

From (2), we know when $\omega=\omega_{1}$ or $\omega=\omega_{2}$, resonance occurs in the system. Resonance frequency of secondary vibration isolation is:

$$
\omega^{2}=\omega_{n}^{2} \frac{1+\mu \pm \sqrt{1+\mu}}{\mu}
$$

if $\omega^{2} / \omega_{n}^{2}=a$, then from (6):

$$
\mu=\frac{2 a-1}{(a-1)^{2}}
$$

Because a is far more than 1, formula (7) can approximately be:

$$
\mu \cong \frac{2}{a-2} \cong \frac{2}{a}
$$

Fig. 1 illustrates equation (8).

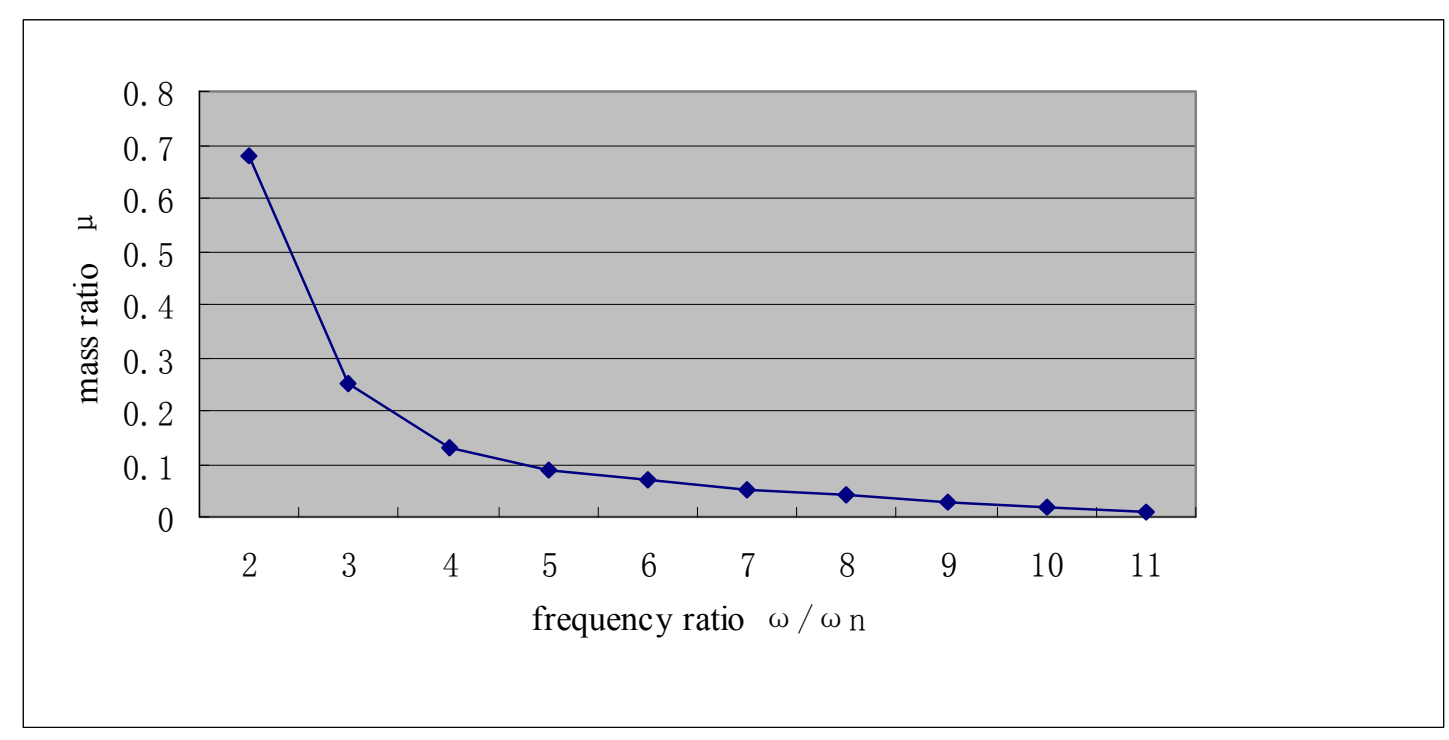

Fig. 1, Relationship of Mass Ratio and Frequency Ratio 
It can be concluded by Fig. 1 that reduction of mass ration changes together with increase of frequency ratio. Therefore, resonance is likely to appear when lighter vibration isolation frame is chosen. The typical frequency ratio of first vibration isolation $\omega / \omega n=3-6$, then mass ratio which causes resonance in the secondary vibration isolation is $0.06-0.25$. To prevent resonance, mass ratio should be more than $0.3^{[8] \text {. }}$

\section{Example Calculation}

Take the secondary vibration isolation of refrigeration compressor unit as an example to research the parameter.

The weight of refrigeration compressor unit is $2800 \mathrm{~kg}$. Frequency modulation working system is available. Revolution adjustment range is $700-2800 \mathrm{r} / \mathrm{min}$.

Six compressors are fixed in a rack shaped like "I". The machine room is located on the ground floor, (the second floor underground exists). In practice, the refrigeration compressor uint is installed in the ceiling. The serious vibration and noise pollute residents on upstairs through sound transmission in solid. Fig. 2 shows the example of refrigeration compressor unit.

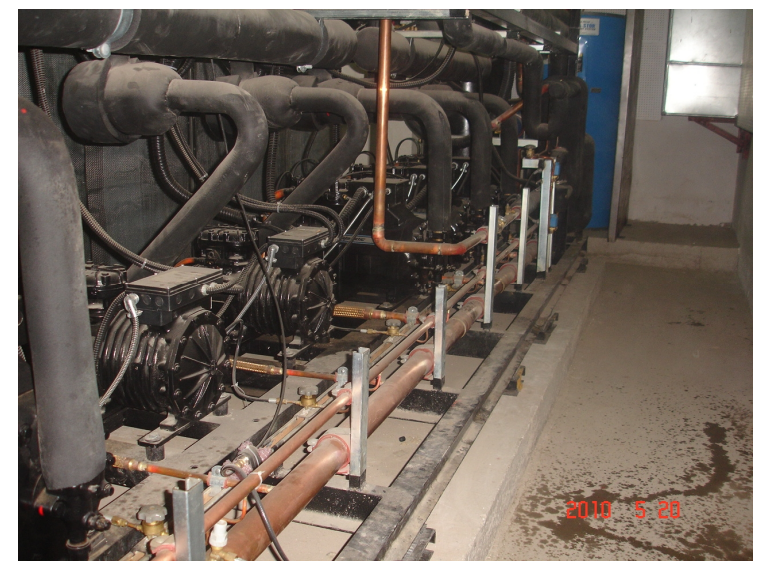

Fig. 2, Photo of Refrigerant Compressor Unit

To abate sound transmission in solid, secondary vibration isolation is adopted. Spring dampers of identical stiffness are prepared for both the upper and the lower layers of the vibration isolation frame. The amount of springs in lower layer will be added in order to maintain consistent deformation of springs along with alteration of mass ratio.

After being calculated by software of excel, Fig. 3, presents the results.

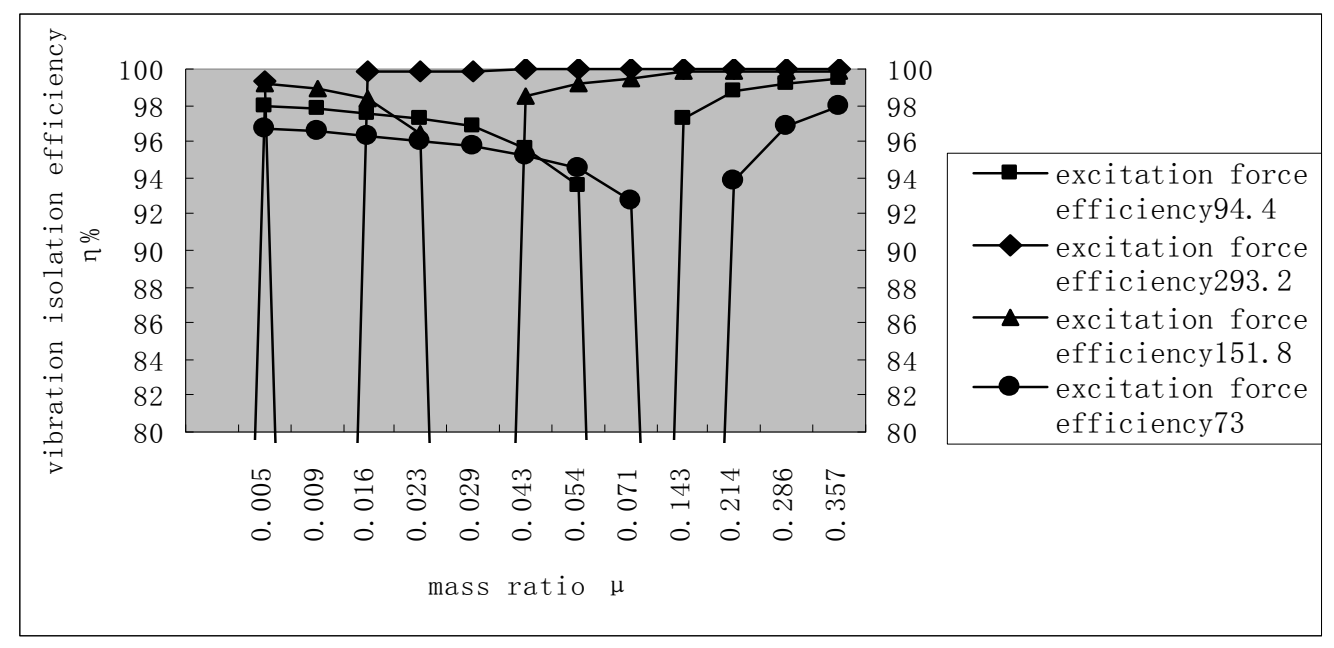

Fig. 3, Relationship between Mass Ration and Vibration Isolation Efficiency 
From Fig. 3, adopting light vibration isolation frame is equivalent to first vibration isolation. The cascading springs will decrease half of the stiffness. According to the theory of first vibration isolation, vibration isolation efficiency can also be relatively high. Meanwhile, from diagram 3, it can be concluded that excitation force efficiency affects mass ratio. The higher the excitation force efficiency is, the lower the mass ratio will be. No resonance of excitation force efficiency can be detected when the mass ratio is above 0.25 . Then the theoretical analysis is verified.

Resonance reflects the negative vibration isolation efficiency. The increased transmitting force and larger amplitude eliminate vibration isolation. Thus, mass ratio is the decisive factor of the project. To avoid resonance range, the appropriate mass ratio is above 0.3 .

\section{Conclusion}

Mass ratio of vibration isolation frame will affect resonance frequency of the system. The resonance frequency can be avoided when the mass ration is more than 0.3 . The larger mass ratio will ensure high efficiency and avoidance of resonance, if possible.

To obtain higher vibration isolation frequency, relatively smaller vibration isolation frame or ratio will be preferable. However, resonance frequency should be avoided when determining mass ratio.

\section{Acknowledgements}

This work was financially funded by the Development Plan Project "Research of Vibration Isolation Technology and Development of New Vibration Isolator" (No: FSKJHT 201352), Fushun Municipal Science and Technology.

\section{References}

[1] Zhiru An, Changlin Han, Application of Secondary Vibration Isolation in Coal Preparation Plant [J].Technique of Coal Preparation.2002.(4):26-27

[2] Nan Xiao, Xin Sun. Application of Secondary Vibration Isolation in Noise Management [J]. Environmental Protection and Recycling Economy.2011(3):59-60

[3] Yan Wen, Futao Zhang, Application of Secondary Vibration Isolation [J]. Education and Science.2014(7):126-127.

[4] Daxian Cheng. Machinery Design, Vol.4 Edition.5 [M]. Beijing: Chemical Industry 2008.

[5] Kecheng Jiang, Application of Secondary Vibration Isolation in Uniaxial Rotating Screen Shaped ZD [J]. Mine Design. 1988(4):19-22

[6] Yuheng Lv. Machines and Material for Noise and Vibration Control [M]. Beijing: Mechanical Industry Press 1999.

[7] Aimin Li, Fei Chen, Xin Lu, Determination of Mass Ratio in Secondary Vibration Isolation Design and Construction of Metallurgical Mine. [J].1995(5):31-33

[8] Dayou Ma. Noise Control [M]. Beijing: Science Press, 1987. 